\title{
Front Matter: Volume 7719
}

, "Front Matter: Volume 7719," Proc. SPIE 7719, Silicon Photonics and Photonic Integrated Circuits II, 771901 (10 June 2010); doi:

10.1117/12.868095

SPIE. Event: SPIE Photonics Europe, 2010, Brussels, Belgium 


\title{
PROCEEDINGS OF SPIE
}

\section{Silicon Photonics and Photonic Integrated Circuits II}

\author{
Giancarlo Cesare Righini \\ Editor
}

12-16 April 2010

Brussels, Belgium

Sponsored by

SPIE

Cosponsored By

B-PHOT_Brussels Photonics Team (Belgium) - Brussels-Capital Region (Belgium) • FWO_Fonds Wetenschappelijk Onderzoek (Belgium) • ICO_International Commission for Optics • Ville de Bruxelles (Belgium)

Cooperating Organisations

CBO-BCO (Belgium) • EOS—European Optical Society (Germany) • IET— The Institution of

Engineering and Technology (United Kingdom) • IOP-Institute of Physics (United Kingdom)

Photonics4Life (Germany) - Photonics@be (Belgium) - Photonics 21 (Germany) • PromOptica (Belgium)

Published by

SPIE

Volume 7719

Proceedings of SPIE, 0277-786X, v. 7719 
The papers included in this volume were part of the technical conference cited on the cover and title page. Papers were selected and subject to review by the editors and conference program committee. Some conference presentations may not be available for publication. The papers published in these proceedings reflect the work and thoughts of the authors and are published herein as submitted. The publisher is not responsible for the validity of the information or for any outcomes resulting from reliance thereon.

Please use the following format to cite material from this book:

Author(s), "Title of Paper," in Silicon Photonics and Photonic Integrated Circuits II, edited by Giancarlo Cesare Righini, Proceedings of SPIE Vol. 7719 (SPIE, Bellingham, WA, 2010) Article CID Number.

ISSN 0277-786X

ISBN 9780819481924

Published by

SPIE

P.O. Box 10, Bellingham, Washington 98227-0010 USA

Telephone +1 3606763290 (Pacific Time) · Fax +1 3606471445

SPIE.org

Copyright (C) 2010, Society of Photo-Optical Instrumentation Engineers

Copying of material in this book for internal or personal use, or for the internal or personal use of specific clients, beyond the fair use provisions granted by the U.S. Copyright Law is authorized by SPIE subject to payment of copying fees. The Transactional Reporting Service base fee for this volume is $\$ 18.00$ per article (or portion thereof), which should be paid directly to the Copyright Clearance Center (CCC), 222 Rosewood Drive, Danvers, MA 01923. Payment may also be made electronically through CCC Online at copyright.com. Other copying for republication, resale, advertising or promotion, or any form of systematic or multiple reproduction of any material in this book is prohibited except with permission in writing from the publisher. The CCC fee code is 0277-786X/10/\$18.00.

Printed in the United States of America.

Publication of record for individual papers is online in the SPIE Digital Library.

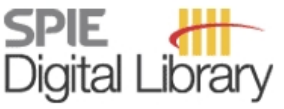

SPIEDigitalLibrary.org

Paper Numbering: Proceedings of SPIE follow an e-First publication model, with papers published first online and then in print and on CD-ROM. Papers are published as they are submitted and meet publication criteria. A unique, consistent, permanent citation identifier (CID) number is assigned to each article at the time of the first publication. Utilization of CIDs allows articles to be fully citable as soon they are published online, and connects the same identifier to all online, print, and electronic versions of the publication. SPIE uses a six-digit CID article numbering system in which:

- The first four digits correspond to the SPIE volume number.

- The last two digits indicate publication order within the volume using a Base 36 numbering system employing both numerals and letters. These two-number sets start with 00, 01, 02, 03, 04 , 05, 06, 07, 08, 09, OA, OB ... 0Z, followed by 10-1Z, 20-2Z, etc.

The CID number appears on each page of the manuscript. The complete citation is used on the first page, and an abbreviated version on subsequent pages. Numbers in the index correspond to the last two digits of the six-digit CID number. 


\section{Contents}

\section{SESSION 1 SILICON PHOTONICS I}

771903 Carrier depletion based silicon optical modulators (Invited Paper) [7719-02]

D. Marris-Morini, G. Rasigade, L. Vivien, Institut d'Electronique Fondamentale, CNRS, Univ. Paris Sud (France); D. J. Thomson, F. Y. Gardes, G. T. Reed, Univ. of Surrey (United Kingdom); J.-M. Fédéli, CEA, LETI, Minatec (France); P. Crozat, E. Cassan, Institut d'Electronique Fondamentale, CNRS, Univ. Paris Sud (France)

$771904 \quad$ Nonlinear silicon photonics (Invited Paper) [7719-03]

K. K. Tsia, Univ. of California, Los Angeles (United States) and Univ. of Hong Kong (Hong Kong, China); B. Jalali, Univ. of California, Los Angeles (United States)

\section{SESSION 2 EUROPEAN PROJECTS IN SILICON PHOTONICS}

771907 HELIOS: photonics electronics functional integration on CMOS (Invited Paper) [7719-06] J.-M. Fédéli, L. Fulbert, CEA, LETI, Minatec (France); D. Van Thourhout, Univ. Gent (Belgium); P. Viktorovitch, I. O'Connor, Institut des Nanotechnologies de Lyon, CNRS, Univ. de Lyon (France); G.-H. Duan, Alcatel-Thales III-V Lab. (France); G. Reed, Univ. of Surrey (United Kingdom); F. Della Corte, Univ. Mediterranea di Reggio Calabria (Italy); L. Vivien, Institut d'Electronique Fondamentale, CNRS, Univ. Paris Sud (France); F. Lopez Royo, Univ. Politécnica de Valencia (Spain); L. Pavesi, Univ. degli Studi di Trento (Italy); B. Garrido, Univ. de Barcelona (Spain); E. Grard, 3S PHOTONICS SA (France); B. Tillack, IHP GmbH (Germany); L. Zimmermann, Technische Univ. Berlin (Germany); S. Formont, Thales Airborne Systems (France); A. Hakansson, DAS Photonics (Spain); E. Wachmann, austriamicrosystems AG (Austria); H. Zimmermann, Technische Univ. Wien (Austria); A. Bakker, PhoeniX B.V. (Netherlands); H. Porte, Photline Technologies (France)

771908 Overview of the EU FP7-project HISTORIC (Invited Paper) [7719-07] G. Morthier, R. Kumar, Univ. Gent (Belgium); F. Raineri, R. Raj, Lab. of Photonics and Nanostructures, CNRS (France); J. Hofrichter, N. Chrysos, B. J. Offrein, IBM Zürich Research GmbH (Switzerland); R. Zhang, J. van der Tol, O. Raz, H. Dorren, Technische Univ. Eindhoven (Netherlands)

771909 The BOOM project: a new generation of photonic routing subsystems using hybrid integration on silicon-on-insulator waveguide boards (Invited Paper) [7719-08] L. Stampoulidis, Constelex Technology Enablers (Greece); K. Vyrsokinos, C. Stamatiadis, H. Avramopoulos, National Technical Univ. of Athens (Greece); L. Zimmermann, Technische Univ. Berlin (Germany) and IHP GmbH (Germany); K. Voigt, Technische Univ. Berlin (Germany); Z. Sheng, D. Van Thourhout, Univ. Gent (Belgium); J. Kreissl, L. Mörl, Fraunhofer-Institut für Nachrichtentechnik, Heinrich-Hertz-Institut (Germany); J. Bolten, T. Wahlbrink, AMO GmbH (Germany); F. Gomez-Agis, E. Tangdiongga, H. J. S. Dorren, Technische Univ. Eindhoven (Netherlands); A. Pagano, E. Riccardi, Telecom Italia (Italy) 
7719 OA The UK silicon photonics project (Invited Paper) [7719-09]

G. T. Reed, N. Wright, G. Z. Mashanovich, B. Timotijevic, Univ. of Surrey (United Kingdom); T. F. Krauss, T. P. White, L. O'Faolain, Univ. of St. Andrews (United Kingdom); R. W. Kelsall, L. Lever, Z. Ikonic, A. Valvanis, Univ. of Leeds (United Kingdom); D. Leadley, E. Findlayson, Univ. of Warwick (United Kingdom); R. M. Jenkins, Malvern Technology Ctr. (United Kingdom)

7719 OB Real-time label-free biosensing with integrated planar waveguide ring resonators (Invited Paper) [7719-10]

H. Sohlström, K. B. Gylfason, D. Hill, KTH-Royal Institute of Technology (Sweden)

\section{SESSION $3 \quad$ PASSIVE PHOTONIC DEVICES}

7719 OC Silicon waveguide-based mode-evolution polarization rotator [7719-11]

J. Zhang, M. YU, G. P. LO, D. L. Kwong, A*STAR Institute of Microelectronics (Singapore)

7719 OE Design, simulation, and fabrication of a $90^{\circ}$ SOI optical hybrid based on the self-imaging principle [7719-13]

S. Abdul-Majid, I. I. Hasan, P. J. Bock, T. J. Hall, Ottawa Univ. (Canada)

7719 OF Highly integrated optical $8 \times 8$ lambda-router in silicon-on-insulator technology: comparison between the ring and racetrack configuration [7719-14]

G. F. Fan, R. Orobtchouk, Institut National des Sciences Appliquées de Lyon (France); J. M. Fédéli, CEA-LETI (France)

7719 OG Characterisation of slab waveguides, fabricated in $\mathrm{CaF}_{2}$ and Er-doped tungsten-tellurite glass by $\mathrm{MeV}$ energy $\mathrm{N}^{+}$ion implantation, using spectroscopic ellipsometry and $\mathrm{m}$-line spectroscopy [7719-15]

I. Bányász, Research Institute for Solid State Physics and Optics (Hungary); S. Berneschi, IFAC-CNR (Italy); T. Lohner, M. Fried, P. Petrik, N. Q. Khanh, Z. Zolnai, Research Institute for Technical Physics and Materials Science (Hungary); A. Watterich, Research Institute for Solid State Physics and Optics (Hungary); M. Bettinelli, Univ. degli Studi di Verona (Italy); M. Brenci, G. Nunzi-Conti, S. Pelli, G. C. Righini, IFAC-CNR (Italy); A. Speghini, Univ. degli Studi di Verona (Italy)

\section{SESSION 4 OPTICAL SOURCES I}

7719 0J Light emission of 2D photonic crystal based on nanocrystal-Si/SiO 2 superlattice structure [7719-18]

M. B. Yu, L. Ding, F.-F. Ren, G. Q. Lo, D. L. Kwong, A*STAR Institute of Microelectronics (Singapore)

\section{SESSION 5 OPTICAL SOURCES II}

7719 OM Silicon nanocrystals light-emitting devices: characterization and coupling to SU-8 waveguides [7719-21]

D. Izquierdo, M. C. Garralaga, I. Salinas, Univ. de Zaragoza (Spain); J. Barreto, C. Domínguez, Ctr. Nacional de Microelectrónica (Spain); I. Garcés, Univ. de Zaragoza (Spain) 
7719 ON Blue and red electroluminescence of silicon-rich oxide light emitting capacitors [7719-22] A. Morales-Sánchez, Instituto Nacional de Astrofísica, Óptica y Electrónica (Mexico) and Ctr. de Nanociencias y Nanotecnología (Mexico); M. Aceves-Mijares,

A. A. González-Fernández, K. Monfil-Leyva, Instituto Nacional de Astrofísica, Óptica y Electrónica (Mexico); J. Juvert, C. Domínguez-Horna, Instituto de Microelectrónica de Barcelona (Spain)

$77190 Q \quad$ Hetero-epitaxial indium phosphide on silicon [7719-25]

C. Junesand, W. Metaferia, F. Olsson, KTH-Royal Institute of Technology (Sweden); M. Avella, J. Jimenez, Univ. de Valladolid (Spain); G. Pozina, L. Hultman, Linköping Univ. (Sweden);

S. Lourdudoss, KTH-Royal Institute of Technology (Sweden)

\section{SESSION 6 OPTICAL DETECTION}

7719 OR Cu/p-Si Schottky photodetectors at 1.55 $\mathrm{\mu m}$ [7719-26]

M. Casalino, M. Gioffrè, G. Coppola, M. Iodice, Institute for Microelectronics and Microsystems (Italy); L. Moretti, Seconda Univ. degli Studi di Napoli (Italy); I. Rendina, L. Sirleto, Institute for Microelectronics and Microsystems (Italy)

7719 OS Hybrid integration of InP photodetectors with SOI waveguides using thermocompression bonding [7719-27]

M. Harjanne, M. Kapulainen, S. Ylinen, T. Aalto, J. Ollila, VTT Technical Research Ctr. of Finland (Finland); L. Mörl, W. Passenberg, Fraunhofer-Institut für Nachrichtentechnik, Heinrich-Hertz-Institut (Germany)

7719 OT Monolithically fabricated germanium-on-SOI photodetector and Si CMOS circuit for integrated photonic applications [7719-28]

K.-W. Ang, T.-Y. Liow, M.-B. Yu, Q. Fang, J. Song, G. Q. Lo, D.-L. Kwong, A*STAR Institute of Microelectronics (Singapore)

7719 OU PIN photodiodes with significantly improved responsivities implemented in a $0.35 \mu \mathrm{m}$ CMOS/BiCMOS technology [7719-29]

I. Jonak-Aver, austriamicrosystems AG (Austria); A. Marchlewski, Technische Univ. Wien (Austria); S. Jessenig, austriamicrosystems AG (Austria); A. Polzer, W. Gaberl, Technische Univ. Wien (Austria); A. Schmiderer, E. Wachmann, austriamicrosystems AG (Austria); H. Zimmermann, Technische Univ. Wien (Austria)

7719 OV Integrated streak camera in standard (Bi)CMOS technology [7719-30]

W. Uhring, J.-P. Le Normand, V. Zint, M. Zlatanski, Lab. InESS (France)

\section{SESSION 7 NONLINEAR PHOTONICS}

7719 OW Silicon-based ultra-wide discrete band conversion (Invited Paper) [7719-31]

O. Boyraz, Univ. of California, Irvine (United States) and Istanbul Sehir Univ. (Turkey); E.-K. Tien, Univ. of California, Irvine (United States); S. Gao, Univ. of California, Irvine (United States) and Zhejiang Univ. (China) 
7719 0X Terahertz-range stimulated emission due to electronic nonlinear frequency conversion in silicon [7719-32]

S. G. Pavlov, Deutsches Zentrum für Luft- und Raumfahrt e.V. (Germany); H.-W. Hübers, Deutsches Zentrum für Luft- und Raumfahrt e.V. (Germany) and Technische Univ. Berlin (Germany); U. Böttger, R. Eichholz, Deutsches Zentrum für Luft- und Raumfahrt e.V. (Germany); V. N. Shastin, Institute for Physics of Microstructures (Russian Federation); N. V. Abrosimov, H. Riemann, Leibniz-Institut für Kristallzüchtung (Germany); H.-J. Pohl, VITCON Projectconsult GmbH (Germany); B. Redlich, FOM-Institute for Plasma Physics Rijnhuizen Netherlands)

7719 OY Enhancing the efficiency of silicon Raman converters [7719-33]

N. Vermeulen, Vrije Univ. Brussel (Belgium); J. E. Sipe, Univ. of Toronto (Canada);

H. Thienpont, Vrije Univ. Brussel (Belgium)

$7719 \mathrm{OZ}$ Strain dependence of second-harmonic generation in silicon [7719-34]

C. Schriever, C. Bohley, J. Schilling, Martin-Luther-Univ. Halle-Wittenberg (Germany);

R. B. Wehrspohn, Martin-Luther-Univ. Halle-Wittenberg (Germany) and Fraunhofer-Institute for Mechanics of Materials (Germany)

\section{SESSION 8 OPTICAL MODULATOR AND SWITCHES}

$771911 \quad$ Hybrid silicon-organic racetrack resonator designs for electro-optical modulation [7719-36] J. Hampe, J. H. Wülbern, S. Prorok, A. Yu. Petrov, M. Eich, Hamburg Univ. of Technology (Germany); J. Luo, A. K.-Y. Jen, Univ. of Washington (United States)

771912 An optimization method for depletion-based silicon optical modulators [7719-37] G. Rasigade, D. Marris-Morini, L. Vivien, E. Cassan, Institut d'Électronique Fondamentale, CNRS, Univ. Paris-Sud (France)

771913 Tunable silicon CROW delay lines [7719-38] F. Morichetti, Fondazione Politecnico di Milano (Italy) and Politecnico di Milano (Italy); A. Canciamilla, M. Torregiani, C. Ferrari, A. Melloni, M. Martinelli, Politecnico di Milano (Italy)

771914 RF frequency transparent $\mathbf{9 0}^{\circ}$ hybrid based on silicon on insulator photonic circuit [7719-39] R. Sambaraju, J. V. Galan-Conejos, J. Herrera, A. Griol, C. Otón, P. Sanchis, A. Martínez, Univ. Politécnica de Valencia (Spain)

\section{SESSION 9 PHOTONIC INTEGRATION}

771916 Cycle-accurate evaluation of reconfigurable photonic networks-on-chip [7719-41] C. Debaes, Vrije Univ. Brussel (Belgium); I. Artundo, Univ. Politécnica de Valencia (Spain); W. Heirman, J. Van Campenhout, Univ. Gent (Belgium); H. Thienpont, Vrije Univ. Brussel (Belgium)

771917 High-speed optoelectronic IC for multi-standards of optical storage system [7719-42] S. Cha, H. Jeong, C. Go, D. Park, C. Lee, K. Kwon, J. Lee, Samsung Electro-Mechanics (Korea, Republic of) 
771918320 Gbps monolithic silicon photonic DWDM receiver [7719-43]

Q. Fang, T.-Y. Liow, K. W. Ang, Y. T. Phang, M. B. YU, G. Q. Lo, D.-L. Kwong, A*STAR Institute of Microelectronics (Singapore)

\section{SESSION 10 WAVEGUIDE AND ACTIVE DEVICES}

7719 1A Rigorous characterization of silicon nanowire for compact nanophotonic devices [7719-45] B. M. A. Rahman, D. M. H. Leung, K. Namassivayane, A. Agrawal, M. Ashraf, H. Tanvir, K. T. V. Grattan, City Univ. London (United Kingdom)

$77191 \mathrm{~B}$ Spatially localized UV-induced crystallization of $\mathrm{SnO}_{2}$ in photorefractive $\mathrm{SiO}_{2}-\mathrm{SnO}_{2}$ thin film [7719-46]

B. N. Shivakiran Bhaktha, Lab. de Physique de la Matière Condensée, CNRS, Univ. de Nice Sophia Antipolis (France); S. Berneschi, G. Nunzi Conti, G. C. Righini, IFAC-CNR (Italy);

A. Chiappini, A. Chiasera, M. Ferrari, IFN-CNR (Italy); S. Turrell, LASIR, CNRS (France) and CERLA, Univ. Lille 1 (France)

7719 1D Low voltage, moderate rejection ratio electro-optic modulator at $2.2 \mu m$ obtained by proton exchange in lithium niobate [7719-48]

O. Caballero-Calero, R. Burla, T. Moulin, A. Delboulbé, L. Jocou, J.-P. Berger, G. Martin, Lab. d'Astrophysique de Grenoble (France)

$77191 \mathrm{E} \quad$ Tunable integrated optical filters based on sapphire microspheres and liquid crystals [7719-49]

G. Gilardi, Consiglio Nazionale delle Ricerche, Istituto per la Microelettronica e Microsistemi (Italy) and Univ. degli Studi di Roma La Sapienza (Italy); H. Yılmaz, M. Sharif Murib, Koç Univ. (Turkey); R. Asquini, Univ. degli Studi di Roma La Sapienza (Italy); A. d'Alessandro, Consiglio Nazionale delle Ricerche, Istituto per la Microelettronica e Microsistemi (Italy) and Univ. degli Studi di Roma La Sapienza (Italy); A. Serpengüzel, Koç Univ. (Turkey); R. Beccherelli, Consiglio Nazionale delle Ricerche, Istituto per la Microelettronica e Microsistemi (Italy)

$77191 \mathrm{~F} \quad$ Electrically driven hybrid Si/III-V lasers based on adiabatic mode transformers [7719-50] B. Ben Bakir, N. Olivier, P. Grosse, S. Messaoudène, S. Brision, E. Augendre, P. Philippe,

K. Gilbert, D. Bordel, J. Harduin, J.-M. Fedeli, CEA, LETI, Minatec (France)

\section{POSTER SESSION}

7719 1G Magnetic nanoparticles-doped silica layer reported on ion-exchanged glass waveguide: towards integrated magneto-optical devices [7719-51]

H. Amata, F. Royer, F. Choveikani, D. Jamon, Univ. de Lyon (France) and Univ. de Saint Etienne (France); J.-E. Broquin, IMEP-LAHC (France); J. C. Plenet, Univ. de Lyon (France) and Lab. de Physique de la Matière Condensée, CNRS, Univ. Lyon 1 (France); J. J. Rousseau, Univ. de Lyon (France) and Univ. de Saint Etienne (France)

$77191 \mathrm{H} \quad$ 10Gbps monolithic silicon FTTH transceiver for PON [7719-52]

J. Zhang, T. Y. Liow, G. Q. Lo, D. L. Kwong, A*STAR Institute of Microelectronics (Singapore)

$771911 \quad$ Laser-assisted chemical etching for texturing silicon surface [7719-53]

M. Saito, S. Kimura, Ryukoku Univ. (Japan) 
$77191 \mathrm{~J}$ Discretely tunable microwave photonics beamformer based on ring resonators and arrayed waveguide gratings [7719-54]

J. D. Doménech, P. Muñoz, J. Capmany, Univ. Politécnica de Valencia (Spain)

$77191 \mathrm{~K}$ Influence of the localization of process-induced disorder on planar photonic crystal waveguide properties [7719-55]

R. Hao, Univ. Paris-Sud 11 (France) and Huazhong Univ. of Science and Technology (China);

E. Cassan, Univ. Paris-Sud 11 (France)

$77191 \mathrm{~L}$ Study on the diffraction performance of the etched blazed grating [7719-56]

S. Li, X. Deng, J. Zhu, Xi'an Jiaotong Univ. (China)

$77191 \mathrm{M} \quad$ Digital holographic microscopy for silicon microsystems metrology [7719-57]

Y. Delacrétaz, C. Depeursinge, Ecole Polytechnique Fédérale de Lausanne (Switzerland)

$77191 \mathrm{~N}$ A 10Gb/s transimpedance amplifier for hybrid integration of a Ge PIN waveguide photodiode [7719-58]

A. Polzer, W. Gaberl, R. Swoboda, H. Zimmermann, Technische Univ. Wien (Austria);

J.-M. Fedeli, CEA, LETI, Minatec (France); L. Vivien, Institut d'Electronique Fondamentale,

CNRS, Univ. Paris Sud XI (France)

7719 is Low-voltage high-efficiency light emitting diodes with lateral-current injection based on truncated $\mathrm{Si}^{-\mathrm{SiO}_{2}}$ quantum wells [7719-63]

L. Ding, M. B. YU, G. Q. LO, D. L. Kwong, A*STAR Institute of Microelectronics (Singapore)

$77191 \mathrm{~T} \quad$ Monolithic integration and optimization of waveguide silicon modulators and germanium photodetectors [7719-64]

T.-Y. Liow, K.-W. Ang, Q. Fang, J. Song, Y.-Z. Xiong, M. YU, G.-Q. Lo, D.-L. Kwong, A*STAR

Institute of Microelectronics (Singapore)

$77191 \mathrm{U} \quad$ Nanometer germanium photodetector with aluminum surface plasmon antenna for enhanced photo-response [7719-65]

F.-F. Ren, K.-W. Ang, G.-Q. LO, D.-L. Kwong, A*STAR Institute of Microelectronics (Singapore)

$77191 \mathrm{~V}$ One theoretical analysis about the two resonators system [7719-66]

G. F. Fan, R. Orobtchouk, Institut National des Sciences Appliquées de Lyon (France)

7719 1W Apodization of coupled resonator optical waveguide devices through a longitudinal offset technique [7719-67]

J. D. Doménech, P. Muñoz, J. Capmany, Univ. Politécnica de Valencia (Spain)

$77191 \mathrm{X}$ Compact integrated optical directional coupler with large cross section silicon waveguides [7719-68]

J. P. George, N. Dasgupta, B. K. Das, Indian Institute of Technology Madras (India)

771912 Design and fabrication of a novel evanescent germanium electro-absorption (EA) modulator [7719-70]

A. E.-J. Lim, K. W. Ang, Q. Fang, T.-Y. Liow, M. Yu, G.-Q. LO, D.-L. Kwong, A*STAR Institute of Microelectronics (Singapore) 
771920 Photonic integrated single-sideband modulator/frequency shifter based on surface acoustic waves [7719-71]

E. C. S. Barretto, J. M. Hvam, Technical Univ. of Denmark (Denmark)

$771921 \quad$ Evolution of black silicon nano- and micro-scale surface topologies upon femtosecond laser irradiation [7719-72]

S. I. Kudryashov, P.N. Lebedev Physical Institute (Russian Federation); E. V. Golosov, Belgorod State Univ. (Russian Federation); A. A. Ionin, P.N. Lebedev Physical Institute (Russian

Federation); Yu. R. Kolobov, Belgorod State Univ. (Russian Federation); A. E. Ligachev, A. M. Prokhorov General Physics Institute (Russian Federation); S. V. Makarov, Yu. N. Novoselov,

L. V. Seleznev, D. V. Sinitsyn, P.N. Lebedev Physical Institute (Russian Federation)

771922 A full-vectorial mode solver for bending waveguides by a modified finite difference method based on E-fields in cylindrical coordinate systems [7719-73]

J. Xiao, X. Sun, Southeast Univ. (China)

771923 Improvement of the channel crosstalk in narrow channel spacing arrayed waveguide gratings applying specially shaped couplers [7719-74]

D. Seyringer, Voralberg Univ. of Applied Sciences (Austria)

Author Index 
Downloaded From: https://www.spiedigitallibrary.org/conference-proceedings-of-spie on 26 Apr 2023

Terms of Use: https://www.spiedigitallibrary.org/terms-of-use 


\title{
Conference Committee
}

\author{
Symposium Chairs
}

Francis Berghmans, Vrije Universiteit Brussel (Belgium)

Ronan Burgess, European Commission (Belgium)

Jürgen Popp, Institut für Photonische Technologien e.V. (Germany)

Peter Hartmann, SCHOTT AG (Germany)

Hugo Thienpont, Vrije Universiteit Brussel (Belgium)

Conference Chair

Giancarlo Cesare Righini, Istituto di Fisica Applicata Nello Carrara (Italy)

Conference Cochairs

Seppo K. Honkanen, Aalto University (Finland)

Bahram Jalali, University of California, Los Angeles (United States)

Lorenzo Pavesi, Università degli Studi di Trento (Italy)

Laurent Vivien, Institut d'Électronique Fondamentale, CNRS, Université de Paris-Sud XI (France)

\section{Program Committee}

Wim Bogaerts, Universiteit Gent (Belgium)

John E. Bowers, University of California, Santa Barbara (United States)

Louay A. Eldada, HelioVolt Corporation (United States)

Jean-Marc Fédéli, CEA, LETI, Minatec (France)

Helmut Heidrich, Fraunhofer-Institut für Nachrichtentechnik, Heinrich-Hertz-Institut (Germany)

Mile Ivanda, Institut Ruder Boškovic (Croatia)

El-Hang Lee, Inha University (Korea, Republic of)

Sebastian Lourdudoss, KTH-Royal Institute of Technology (Sweden)

Mario J. Paniccia, Intel Corporation (United States)

Thomas P. Pearsall, European Photonics Industry Consortium (France)

Stefano Pelli, Istituto di Fisica Applicata Nello Carrara (Italy)

Klaus Petermann, Technische Universität Berlin (Germany)

Stavros Pissadakis, Foundation for Research and Technology-Hellas (Greece)

Francesco Priolo, Università degli Studi di Catania (Italy)

Manijeh Razeghi, Northwestern University (United States)

Juha T. Rantala, Silecs Oy (Finland)

Graham T. Reed, University of Surrey (United Kingdom) 
Ali Serpengüzel, Koç University (Turkey)

Luigi Sirleto, Istituto per la Microelettronica e Microsistemi (Italy)

Ari Tervonen, Helsinki University of Technology (Finland)

Brian R. West, Wilfrid Laurier University (Canada)

Session Chairs

$1 \quad$ Silicon Photonics I

Lorenzo Pavesi, Università degli Studi di Trento (Italy)

2 European Projects in Silicon Photonics

Laurent Vivien, Institut d'Électronique Fondamentale, CNRS, Université de Paris-Sud XI (France)

3 Passive Photonic Devices

Seppo K. Honkanen, Aalto University (Finland)

4 Optical Sources I

Maurizio Ferrari, IFN-CNR (Italy)

5 Optical Sources II

Stefano Pelli, Istituto di Fisica Applicata Nello Carrara (Italy)

6 Optical Detection

Jean-Marc Fédéli, CEA, LETI, Minatec (France)

$7 \quad$ Nonlinear Photonics

Luigi Sirleto, Istituto per la Microelettronica e Microsistemi (Italy)

8 Optical Modulator and Switches

Giancarlo Cesare Righini, Istituto di Fisica Applicata Nello Carrara (Italy)

9 Photonic Integration

Jean-Marc Fédéli, CEA, LETI, Minatec (France)

10 Waveguide and Active Devices

Jean-Marc Fédéli, CEA, LETI, Minatec (France) 